\title{
Detección de enfermedades en cultivos de papa usando procesamiento de imágenes
}

\section{Disease detection in Potato crops using image processing}

\author{
Rosero Acosta Noreen Odalys \\ Universidad Politécnica Estatal del Carchi.(Ecuador) \\ noreen.rosero@upec.edu.ec
}

Cabrera Rosero Jairo Andrés Universidad Politécnica Estatal del Carchi.(Ecuador) jairo.cabrera@upec.edu.ec

Anrango Oyagata Malkik Salvador Universidad Politécnica Estatal del Carchi.(Ecuador) malkik.anrango@upec.edu.ec

Yandún Velasteguí Marco Universidad Politécnica Estatal del Carchi.(Ecuador) marco.yandun@upec.edu.ec

Lascano Rivera Samuel Universidad Politécnica Estatal del Carchi.(Ecuador) samuel.lascano@upec.edu.ec

\section{Revista Cumbres Vol.6 №1}

Versión electrónica ISSN 1390-3365 http://investigacion.utmachala.edu.ec/revistas/index.php/Cumbres 


\title{
RESUMEN
}

El presente trabajo describe las técnicas y procesos empleados en un sistema de identificación y clasificación de enfermedades en cultivos de papa usando procesamiento de imágenes que permite determinar las fases que presenta la Alternariosis una enfermedad muy común en el cultivo de papa, pero debido a los diferentes tipos de enfermedades presentes en la hoja de papa, se aplicó además para determinar los tres tipos de plagas más comunes en el cultivo: Alternariosis (Alternaria solani), Tizón Tardío (Phytophthora infestans) y Virosis (PVS). Este tipo de enfermedades causan un daño notorio en la hoja de la planta, una de las ventajas más importantes para el sistema de detección por medio del procesamiento de imágenes, se resalta la importancia de utilizar un sistema para la detección temprana de enfermedades en los cultivos por parte de los agricultores, se describen los métodos y procesos utilizados para la detección de las enfermedades en las hojas de papa, métodos presentes en cinco pasos fundamentales al momento de la detección y clasificación de un objeto dentro de una imagen que en su lugar está conformado por una imagen de una hoja de papa enferma, como primer paso se realiza la adquisición de imagen y finaliza con la clasificación de la enfermedad usando la red neuronal ANN (Artificial Neural Networks). Por último, se presenta los resultados obtenidos usando el sistema de detección de enfermedades presentes en la hoja de papa por medio del procesamiento de imágenes, usando el aplicativo Matlab

Palabras clave: Agro-tecnología, cultivo de papa, detección de imágenes.

\begin{abstract}
This work describes the techniques and processes used in a system of identification and classification of diseases in potato crops. They use image processing that allows to determine the phases that Alternariosis presents a very common disease in potato cultivation. Due to the different types of diseases present in the potato leaf, Alternariosis (Alternaria solani), Late blight (Phytophthora infestans) and Virosis (PVS) were also applied to determine the three most common types of pests in the crop. This type of disease causes a noticeable damage to the plant leaf, one of the most important advantages for the detection system through image processing. The importance of using a system for the early detection of diseases in crops is highlighted by farmers. The methods and processes used for the detection of the diseases in the potato leaves are described. These methods are present in five fundamental steps at the time of the detection and classification of an object within an image of a sick potato leaf. As a first step, the image is acquired and it
\end{abstract}

\section{Cumbres}


ends with the classification of the disease using the ANN (Artificial Neural Networks) neural network. Finally, the results are presented by using the disease detection system in the potato leaf through image processing, using the Matlab application.

Keywords: Agro-technology, potato cultivation, image detection.

\section{INTRODUCCIÓN}

La tecnología está inmersa en casi todas las áreas o ciencias en las cuales se tiene como objetivo resolver y facilitar un determinado problema o trabajo. En este mismo sentido, su aplicación se presencia en el sector agrícola, pues ha servido históricamente como herramienta entre el hombre y la naturaleza, ambos agentes marcan un proceso de cambio tecnológico y de innovación, las cuales impactan directamente en la producción (Olivares, Castro, Miranda, Morales, y Barrera, 2019).

Los términos agricultura y tecnología forman un estudio el cual hoy en día se conoce como agro tecnología, puesto a que abarca dos conocimientos muy amplios. Sin embargo, lo que se trata es especificar o enfocar esta temática en una sola, como puede ser la producción de la papa Al-Gaadi, Khalid A., et al. (2016).

De acuerdo a Ronco et al. (2009): "La región de los Andes Centrales de Perú, Ecuador y Bolivia tiene una riqueza única de sistemas de producción de papa. Por miles de años los agricultores de zonas alto-andinas localizadas entre los 3500 y 4200 m sobre el nivel del mar, han desarrollado tecnologías tradicionales adaptadas a condiciones climáticas severas."

Pérez, Méndez, Pérez, Elías y Corimayo (2014) aseguran: "El procesamiento digital de imágenes es un campo fascinante que incluye varias ciencias, tales como óptica, electrónica, matemática, fotografía e informática" (p.724). Utilizar esta agrupación de ciencias para la detección temprana de enfermedades en el cultivo de papa y que esta detección sea precisa y confiable, le da la oportunidad al agricultor de encontrar una nueva estrategia para combatir las plagas sin necesidad de utilizar diferentes tipos de químicos que a final de cuentas va a afectar al tubérculo, a la salud del agricultor y consumidor.

\section{MATERIALES Y METODOS}

Los seres vivos detectamos y reconocemos formas, objetos, colores, contornos en imágenes con facilidad. Esta tarea no es complicada en nuestro entorno, pero si se presenta una gran dificultad para los sistemas de visión por computadora, ya que estos no cuentan con un proceso de inteligencia artificial que cumpla la visión de una persona. Generalmente, un sistema de 
reconocimiento de objetos está diseñado para encontrar e identificar objetos específicos en una imagen es decir identifica cualquier tipo de objetos dentro de una imagen.

Una gran cantidad de problemas originados en diferentes campos como las industrias, seguridad, medicina, robótica, se han resuelto mediante la combinación de diversas técnicas de procesamiento de imágenes y detección de patrones. Miranda, Johnny L., et al. (2014), para el reconocimiento y detección de objetos comúnmente se sigue un proceso conformado por cinco pasos, cada uno de estos pasos son muy importantes al momento de la detección y clasificación de un objeto dentro de una imagen, estos pasos son los que se cumple para la detección y clasificación de enfermedades en plantas usando el procesamiento de imágenes.

\section{Adquisición de imagen}

Las imágenes de la hoja de la planta se capturan a través de la cámara para su procesamiento, almacenamiento y transmisión. Esta imagen está en forma RGB (rojo, verde y azul) imagen a color.

\section{Pre procesamiento de imagen}

Se aplica técnicas de mejoramiento de contraste, reducción de ruido, realce de características, estas técnicas se aplican con la finalidad de eliminar cualquier otro objeto que no sea necesario en la imagen, el recorte de imagen es muy importante para obtener la región de la imagen interesada.

El suavizado de imagen se realiza utilizando el filtro de suavizado. La mejora se lleva a cabo para aumentar el contraste. Luego, la ecualización del histograma que distribuye la intensidad de la escala de grises para mejorar las imágenes de la enfermedad de las plantas.

\section{Segmentación de imagen}

La segmentación significa la división de la imagen en varias partes de mismas características o que tengan alguna similitud. La segmentación se puede hacer usando varios métodos como el método Otsu, k-means, agrupación, conversión de imagen RGB en su modelo HIS, etc.

- Segmentación con algoritmo de detección de límites y puntos: La imagen RGB se convierte en el modelo HIS para la segmentación. La detección de límites y la detección puntual ayudan a encontrar la parte infectada de la hoja. Según García, (2015) el modelo HSI los colores se representan mediante sus propiedades: Tono, Saturación e Intensidad (HSI: Hue, Saturation, Intensity), que describen color, pureza del color y brillo. El tono equivale al color que se aprecia. La saturación se refiere a la pureza de dicho color (su grado de mezcla con los otros colores primarios. Por último, la intensidad puede identificarse como el brillo de la imagen. 
- K-means clustering: La agrupación K-means se usa para clasificar objetos basado en un conjunto de características en $\mathrm{K}$ número de clases. La clasificación del objeto se realiza minimizando la suma de cuadrados de la distancia entre el objeto y el grupo correspondiente.

Algoritmo de umbral de Otsu: García, (2015) menciona que la importancia del método de Otsu radica en que es automático, es decir, no necesita supervisión humana ni información previa de la imagen antes de su procesamiento. Este método se emplea cuando hay una clara diferencia entre los objetos a extraer respecto del fondo de la escena. En este método, se elige el umbral óptimo maximizando la varianza entre clases mediante una búsqueda exhaustiva. A medida que el número de clases de una imagen aumenta, el método de Otsu necesita mucho más tiempo para seleccionar un umbral multinivel adecuado. La creación de umbrales crea imágenes binarias a partir de imágenes en nivel de gris para establecer todos los píxeles por debajo de un umbral a cero y todos los píxeles por encima de ese umbral a uno.

La hoja infectada muestra los síntomas de la enfermedad al cambiar el color de la hoja. El verde de las imágenes de las hojas se puede usar para detectar la porción infectada de la hoja. Los componentes R, G y B se extraen de la imagen. El umbral se calcula utilizando el método de Otsu. Luego, los píxeles verdes se enmascaran y se eliminan y las intensidades de píxeles son inferiores al umbral calculado.

\section{Extracción de características}

La extracción de características juega un papel importante para la identificación de un objeto. Se utiliza la extracción de color, la textura, la morfología, los bordes, entre otros.

Estas son las características más relevantes que se pueden utilizar en la detección de enfermedades de las plantas. La textura significa cómo se distribuye el color en la imagen, la aspereza, la dureza de la imagen, características las cuales son utilizadas para la detección de áreas de plantas infectadas.

\section{Clasificación usando la red neuronal ANN (Artificial Neural Networks)}

Después de realizar la extracción de características, las imágenes de la base de datos de aprendizaje se clasifican mediante el uso de la red neuronal. Estos vectores de características se consideran neuronas en ANN. La salida de la neurona es la función de la suma ponderada de las entradas.

En este caso las características de la imagen entran en comparación con los datos almacenados en la base de datos conformada por imágenes de hojas enfermas y la imagen de entrada posteriormente analizada y de la misma forma con sus características principales extraídas, la red neuronal clasifica 
a que rango pertenece y se asemeja al tipo de enfermedad, con la finalidad de arrojar resultados exactos. Entre mayor sea el peso de datos almacenado en la red de aprendizaje los resultados serán más precisos.

\section{DISCUSIÓN Y RESULTADOS}

\section{Adquisición de imagen}

Para la adquisición de imágenes se realizó una investigación de las diferentes plagas que afectan a los cultivos de papa en la zona Andina, obteniendo como resultado la selección de las siguientes enfermedades para ser analizadas:

Alternariosis: Martínez, Barrios, y Santos (2007) asegura: “Alternaria, o Tizón Temprano es un hongo que se caracteriza por las manchas circulares con anillos concéntricos de color pardo oscuro. La infección comienza por las hojas más viejas y pueden extenderse a todo el follaje, causando la defoliación total de la planta, las hojas fuertemente atacadas se tornan amarillas y se caen" (pp.221-222).

Figura 1. Alternaria en la hoja de la papa

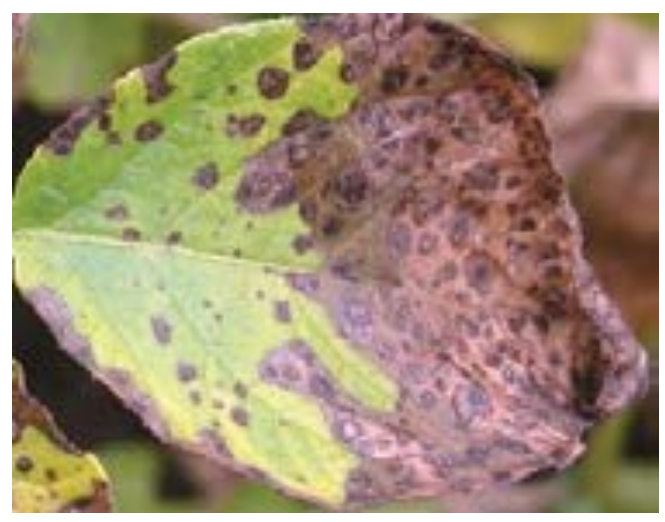

Tizón Tardío: Martínez, Barrios, y Santos (2007) afirma: "El ciclo de este hongo es bastante complejo, con la formación de varios tipos de esporas, el desarrollo de la enfermedad está favorecido por la humedad. Es la plaga más peligrosa y destructiva ya que, al presentarse condiciones favorables para su desarrollo, puede en pocas horas causar la defoliación y muerte de las plantas" (pp.219-221).

Figura 2. Tizón tardío en la hoja de la papa.

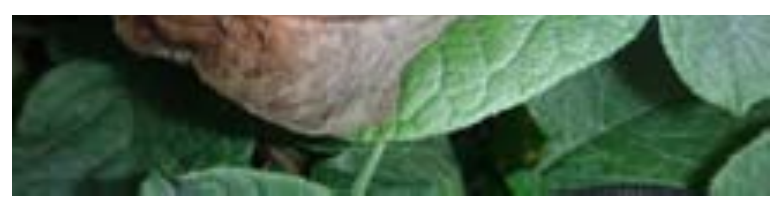

Virosis: Virus que causa enanismo, amarillamiento, deformación de las hojas y necrosis tanto en las hojas como en el tubérculo (Pérez y Forbes, 2011, p.11). Además, Acuña y Sandoval (2017) señalan: "Su importancia radica en

\section{Cumbres}


que genera pérdidas importantes en el rendimiento y calidad del cultivo, además de un aumento en el rechazo de semilleros para certificación" (p.1).

Figura 3.Virosis en la hoja de la papa

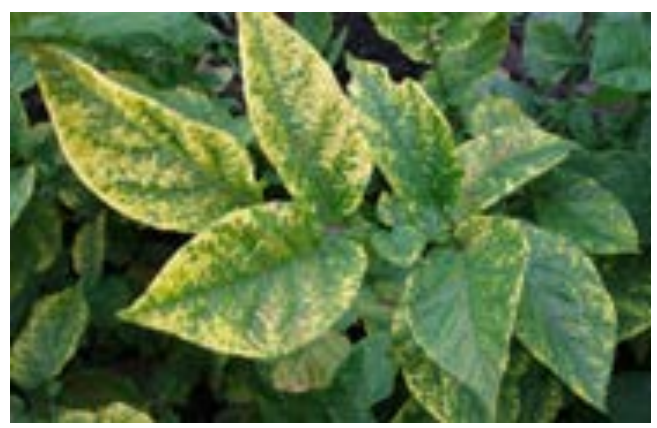

\section{Pre procesamiento de imagen}

En la Figura 4 se puede observar cómo mediante las diferentes técnicas utilizadas en la imagen adquirida dan realce a las características de la misma, teniendo como resultado una imagen con un contraste mejorado.

Figura 4. Imagen con contraste aumentado

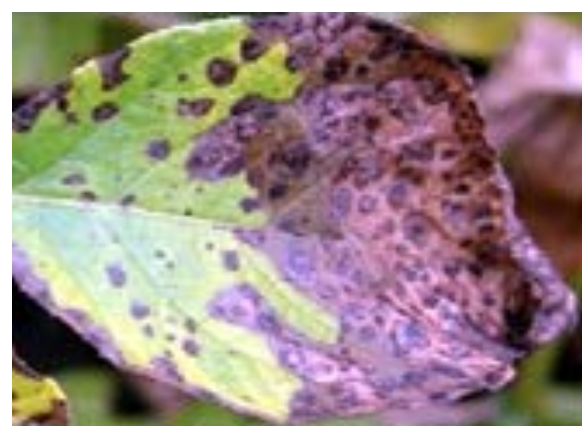

\section{Segmentación de imagen}

La segmentación de la imagen obtuvo los siguientes resultados, en los que se puede notar como cada tipo de segmentación le da un aspecto diferente a la imagen haciendo resaltar la enfermedad.

Segmentación con algoritmo de detección de límites y puntos.

Figura 5. Imagen segmentada con algoritmo de detección de límites y puntos

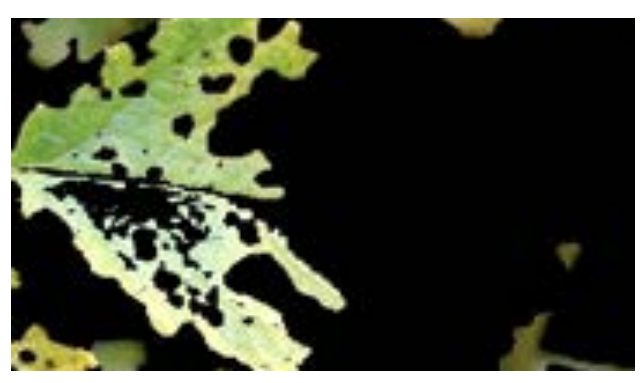


Figura 6. Imagen Segmentada con K-means clustering

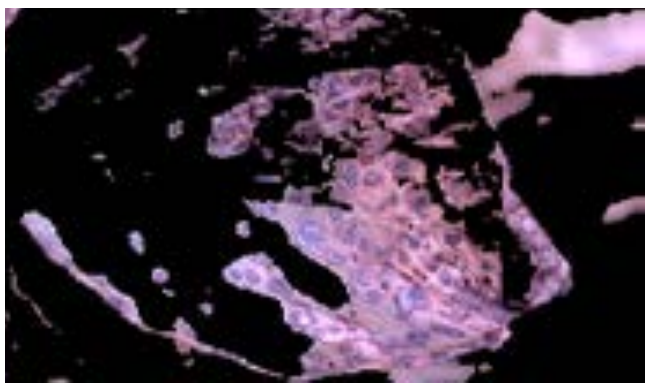

Figura 7. Imagen segmentada con el algoritmo de umbral de Otsu

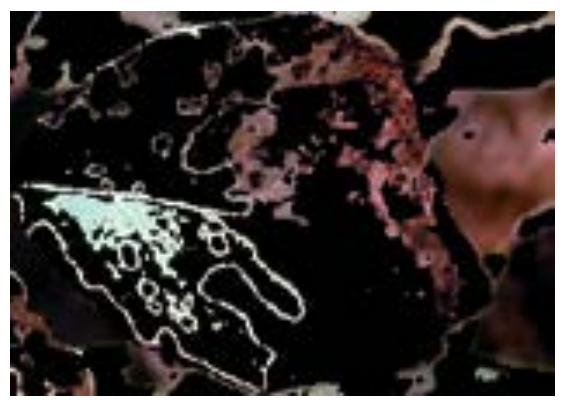

\section{Extracción de características}

La extracción de características dio como resultado 14 datos, que corresponden a la imagen adquirida para la detección de la enfermedad.

Figura 8. Datos extraídos de la imagen.

\begin{tabular}{|c|c|c|c|c|c|c|c|c|c|}
\hline & 1 & 2 & 3 & 4 & 3 & 6 & 7 & 1 & 9 \\
\hline 1. & 애영 & जाष & LXW & Distes & MLES & stait & LTs & 5574 & $2187 t+6$ \\
\hline 2 & CQ58 & 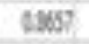 & $2 \times ?$ & 0.585 & K1SN & a116 & tw5s & $49 x$ & $1020 t+03$ \\
\hline 3 & 0.065 & asise & $0 \times 57$ & 0868 & 16 ant & Stated & 1659 & syes & $200 \mathrm{sil} t+0 \mathrm{I}$ \\
\hline 4 & 05412 & 27510 & 25502 & QQN2 & 10971 & 375015 & 2500 & 2a:37 & $13 \times 6 k+0.9]$ \\
\hline 5 & 0598 & ariet & OAde? & Q.5Tा & 17.115 & 3sses & 2802 & wass & $1042 t+63$ \\
\hline & ane & $\ldots$ & $\therefore$ & anes & natat & Hasa & $\ldots$ & N.t. & ante a \\
\hline
\end{tabular}

\section{Clasificación usando la red neuronal ANN (Artificial Neural Networks)}

La comparación entre los datos extraídos de la imagen y la base de datos que consta de las tres enfermedades escogidas, arroja como resultado la enfermedad que tiene la hoja analizada.

\section{Cumbres}


Figura 9. Resultado de la comparación de la imagen ingresada

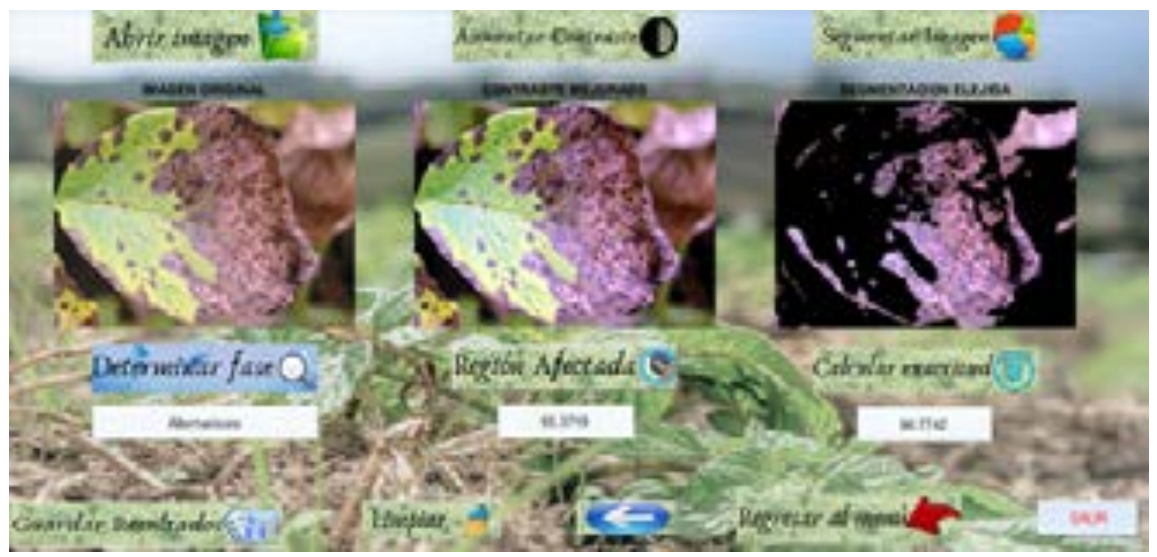

\section{CONCLUSIONES}

La detección de los diferentes tipos de enfermedades que se presentan en los cultivos de papa durante los procesos de desarrollo, es de gran relevancia ya que la detección oportuna de una plaga en etapa temprana ahorra tiempo y costos al momento de aplicar el tratamiento necesario, generando productos sanos para el consumo y reducción de la contaminación por el uso excesivo de fungicidas.

Además, existen varias técnicas para segmentar la parte de la enfermedad de la planta, extracción y clasificación de características para identificar los rasgos principales de las hojas infectadas y la clasificación de enfermedades de las plantas, técnicas que al ser combinadas por medio del procesamiento de imágenes se puede obtener una detección exacta de las diferentes enfermedades que están presentes en los cultivos.

También, el uso de métodos como redes neuronales de aprendizaje ANN para la clasificación de enfermedades en plantas, como el mapa de características de auto organización, el algoritmo de propagación inversa, SVM, etc., puede usarse de manera eficiente. A partir de estos métodos, podemos identificar y clasificar con precisión diversas enfermedades de las plantas utilizando técnicas de procesamiento de imágenes.

Y finalmente se puede asegurar que la aplicación de herramientas tecnológicas es un factor de desarrollo en los procesos que involucran la siembra y cosecha de cultivos de papa, detonante para la evolución del trabajo y por consiguiente resultados óptimos para el sector agrícola.

\section{REFERENCIAS BIBLIOGRÁFICAS}

Acuña, I. y Sandoval, C. (2017). Virología - Virus en papa, sanidad vegetal recuperado de: http://www.inia.cl/wp-content/uploads/FichasTecnicasSanidadVegetal/Ficha\%2050\%20Virosis\%20de\%20la\%20papa.pdf 
Al-Gaadi, Khalid A., et al. "Prediction of Potato Crop Yield Using Precision Agriculture Techniques." PLOS ONE, vol. 11, no. 9, 2016.

García, E. (2015). Detección y clasificación de objetos dentro de un salón de clases empleando técnicas de procesamiento digital de imágenes. Ciudad de México, México.

Martínez, Barrios, y Santos. (2007). Manejo Integrado de Plagas. Tarragona, España: Editorial Grup Bou. Recuperado de Manejo Integrado de Plagas, pp.119-221.

Miranda, Johnny L., et al. "Pest Detection and Extraction Using Image Processing Techniques." International Journal of Computer and Communication Engineering, vol. 3, no. 3, 2014, pp. 189-192.

Olivares, A., Castro, E., Miranda, P., Morales, F., y Barrera, C. (enero de 2019). Competitividad Organizacional: Estudio de Factores. Recuperado de: https://www.academia.edu/38470015/Competitividad_Organizacional_Estudio_de_Factores

Pérez y Forbes. (2011). Guía de Identificación de Plagas que Afectan a la papa en la Zona Andina. Lima, Perú: Centro Internacional de la papa (CIP), p.11.

Pérez, M., Méndez, S., Pérez, N., Elías, R., y Corimayo, R. (2014). Restauración de imágenes basadas en metaheurísticas y entornos paralelos. WICC 2014 XVI Workshop de Investigadores en Ciencias de la Computación, p.724.

Ronco, C., Arias, M., Oswaldo, Estrella, E., Rivera, Yumisaca, y Piedra, A. (2009). Tecnologías sostenibles y su uso en la producción de papa en la región alto andina. Revista Latinoamericana de la papa, p.37. 